EPJ Web of Conferences 60, 20031 (2013)

DOI: $10.1051 /$ epjconf/20136020031

(C) Owned by the authors, published by EDP Sciences, 2013

\title{
Search for an excited quark decaying in semi-leptonic channel
}

\author{
Ta-Wei Wang ${ }^{1, a}$ (on behalf of the CMS Collaboration) \\ ${ }^{1}$ National Taiwan University, Taipei, Taiwan.
}

\begin{abstract}
A search is performed for a pair-produced excited top quark, $\mathrm{t}^{*}$, that decays to a top quark and a gluon using data collected by the CMS detector from pp collisions at $\sqrt{s}=8 \mathrm{TeV}$. The search is performed using events that have a single isolated muon or electron, missing transverse momentum, and at least six jets, one of which must be identified as originating from the fragmentation of a b quark. The data analyzed correspond to an integrated luminosity of $19.6 \mathrm{fb}^{-1}$. No significant excess is observed and we set a lower limit on the $\mathrm{t}^{*}$-quark mass of $790 \mathrm{GeV} / c^{2}$ at $95 \%$ confidence level.
\end{abstract}

\section{Introduction}

The top quark, discovered in 1995, with its large mass of $173.5 \pm 0.6 \pm 0.8 \mathrm{GeV} / c^{2}[1]$, is unique among the fundamental particles. Many theories have emerged surmising that it may be a composite particle rather than an elementary particle. A direct test of this possibility would be to show the existence of an excited top quark $\left(\mathrm{t}^{*}\right)$.

In this analysis, we adopt a model in which the $\mathrm{t}^{*}$ quark has spin $3 / 2$ and decays predominantly to a top quark via the emission of a gluon $[2,3]$. Such a heavy spin-3/2 excitation of a heavy spin-1/2 quark is governed by the RaritaSchwinger [4] vector spinor Lagrangian and the rate of production for such a spin-3/2 quark is higher than for a similarly massive spin-1/2 quark.

In string realizations of the Randall-Sundrum (RS) model, the right-handed $\mathrm{t}^{*}$ quark is expected to be the lightest spin-3/2 Regge excitation [2]. The pair-production cross section at $\sqrt{s}=8 \mathrm{TeV}$ has been calculated to be of the order of a few pb for $m_{\mathrm{t}^{*}}=500 \mathrm{GeV} / c^{2}$ [3].

For this analysis, we assume a $100 \%$ branching fraction for $\mathrm{t}^{*} \rightarrow \mathrm{tg}$, since this decay channel dominates over others. Since mixing between spin- $1 / 2$ and spin-3/2 states is suppressed, we consider only pair production of the $\mathrm{t}^{*}$ quark and its antiparticle in this analysis. We consider decay channels having a single lepton in the final state, either a muon or an electron.

\section{CMS Detector and Data Samples}

The central feature of the Compact Muon Solenoid (CMS) apparatus is a superconducting solenoid of $6 \mathrm{~m}$ internal diameter, providing a magnetic field of $3.8 \mathrm{~T}$. Within the superconducting solenoid volume are a silicon pixel and strip tracker, a lead tungstate crystal electromagnetic calorimeter (ECAL), and a brass/scintillator hadron calorimeter

\footnotetext{
ae-mail: ta-wei.wang@cern.ch
}

(HCAL). Muons are measured in gas-ionization detectors embedded in the steel return yoke outside the solenoid. Extensive forward calorimetry complements the coverage provided by the barrel and endcap detectors. A more detailed description can be found in [5].

The data used in this analysis were recorded during 2012 by the CMS detector at the LHC, utilizing pp collisions at $\sqrt{s}=8 \mathrm{TeV}$. The data analyzed correspond to an integrated luminosity of $19.6 \mathrm{fb}^{-1}$ and were acquired using triggers that require at least one lepton candidate and at least three central jets

Simulated $\mathrm{t}^{*} \mathrm{t}^{*}$ signal events, including up to two additional hard partons, are generated for $\mathrm{t}^{*}$ using the MADGRAPH 5 [6] event generator with CTEQ611 [7] parton distribution functions (PDFs). Generated events are processed through the CMS detector simulation based on GeANT4 4.3.1 [8].

\section{Event Selection}

The selection for the $\mu+$ jets channel requires exactly one muon with $p_{\mathrm{T}}>26 \mathrm{GeV} / c,|\eta|<2.1$, relative isolation $<$ 0.12 , and a transverse impact parameter (longitudinal distance) with respect to the primary vertex $<2 \mathrm{~mm}(<$ $5 \mathrm{~mm}$ ). The e+jets selection requires exactly one electron having $p_{\mathrm{T}}>30 \mathrm{GeV} / c,|\eta|<1.5$, relative isolation $<0.1$, and an impact parameter with respect to the primary vertex transverse to the beam direction $<0.2 \mathrm{~mm}$. The trigger requirements described in Sec. 2 define the thresholds for the lepton $p_{\mathrm{T}}$.

Events with exactly one isolated lepton and at least six jets with $p_{\mathrm{T}}>30 \mathrm{GeV} / c$ and $|\eta|<2.5$ are selected. To meet trigger requirements, the leading three jets are required to have transverse momenta of $p_{\mathrm{T}}>45 \mathrm{GeV} / c$ in the early data taking period and $p_{\mathrm{T}}>55 \mathrm{GeV} / c, p_{\mathrm{T}}>45 \mathrm{GeV} / c$, and $p_{\mathrm{T}}>35 \mathrm{GeV} / c$, respectively, at later times. At least one jet must be b-tagged. 
After imposing our event selection we observe a total of 13636 events in the $\mu+$ jets channel and 11643 events in the e+jets channel.

\section{Mass Reconstruction}

The procedure adopted for reconstructing the mass is described as follows: after assigning the reconstructed objects to the partons from the decays we perform a kinematic fit to improve the resolution of the reconstructed mass of the $t^{*}$ candidates. In the lepton+jets channel, one $\mathrm{W}$ boson decays leptonically, while the other $\mathrm{W}$ boson decays hadronically, resulting in the final state:

$$
\mathrm{t}^{*} \mathrm{t}^{*} \rightarrow(\ell v \mathrm{bg})(\mathrm{q} \overline{\mathrm{q}} \mathrm{bg})
$$

The momenta of the particles in the final state must satisfy the following constraints:

$$
\begin{aligned}
m(\ell v)=m(\mathrm{q} \overline{\mathrm{q}}) & =M_{\mathrm{W}} \\
m(\ell \nu \mathrm{b})=m(\mathrm{q} \overline{\mathrm{q}}) & =M_{\mathrm{t}} \\
m(\ell v \mathrm{bg})=m(\mathrm{q} \overline{\mathrm{q}} \mathrm{gg}) & =M_{\mathrm{t}+\mathrm{g}},
\end{aligned}
$$

where $M_{\mathrm{W}}=80.4 \mathrm{GeV} / c^{2}$ is the mass of the $\mathrm{W}$ boson, $M_{\mathrm{t}}=$ $173.5 \mathrm{GeV} / c^{2}$ is the mass of the top quark, and $M_{\mathrm{t}+\mathrm{g}}$ is a free parameter that is optimized in the fit.

The reconstructed objects in the event that are assigned to a parton for the fit are the charged lepton, the missing transverse momentum $p_{\mathrm{T}}$, and the six leading jets. With one unknown and five constraints we perform a kinematic fit by minimizing a $\chi^{2}$ computed from the difference between the measured momenta of all final-state particles and their fitted values, divided by the measurement uncertainty, subject to the kinematic constraints listed above.

All permutations of jet-quark assignments are considered, subject to the condition that a b-tagged jet must be assigned to one of the $b$ quarks (if multiple jets are tagged, two of them are assigned as b quarks). The jet-quark assignment with the smallest $\chi^{2}$ value is chosen for reconstructing the event. Figure 1 shows the distribution of the reconstructed top plus gluon mass, $M_{\mathrm{t}+\mathrm{g}}$ for the $\mu+\mathrm{jets}$ and e+jets channels.

\section{Background Estimation}

We use a data-driven method to estimate the background contribution to the signal region. We model the background from standard model sources using a Fermi-like function:

$$
f(x)=\frac{a}{1+e^{\frac{x-b}{c}}},
$$

where $x$ represents the reconstructed mass and $a, b$, and $c$ are parameters that are determined by a fit to the data. The $\mathrm{t}^{*}$-signal distribution is modeled using simulated samples. Figure 1 shows the fit to the reconstructed top+gluon mass distribution using Eq. 4.

We fit the background function plus the signal model to the reconstructed mass spectrum observed in data above a mass of $350 \mathrm{GeV} / c^{2}$ (the function describes the tail of the background distribution, not the peak at low mass). The
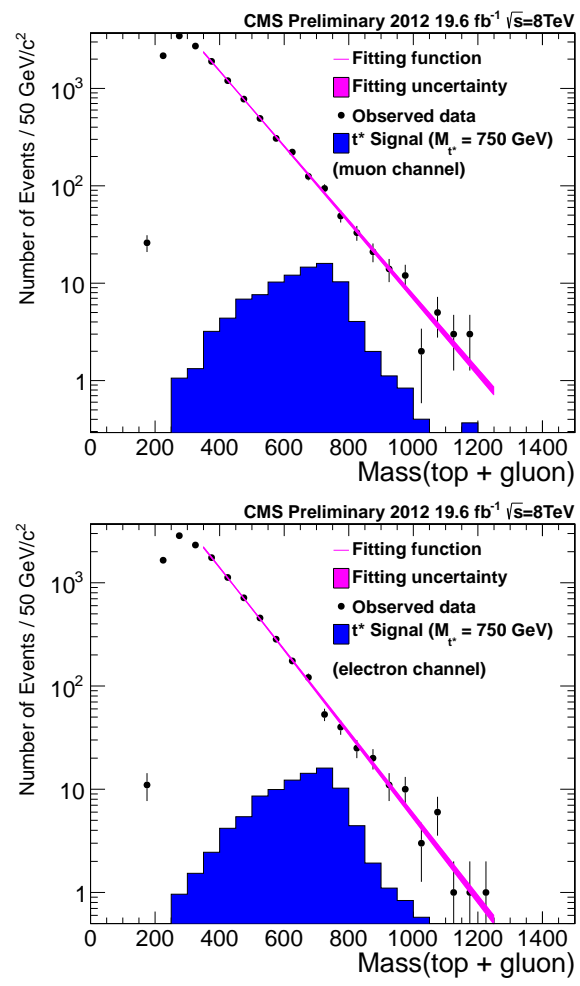

Figure 1. The reconstructed top+gluon mass spectrum for data (points) along with the background fit to the data (curve), and signal distribution (histogram) in the $\mu+$ jets (up) and e+jets (down) channels. The reconstructed masses are those of the jet-quark assignment with the smallest $\chi^{2}$ value for each event.

three parameters of the background function and the signal cross section are allowed to float during the maximum likelihood fit.

\section{Systematic Uncertainties}

Systematic uncertainties influence the signal and background predictions for the $M_{\mathrm{t}+\mathrm{g}}$ distribution that are used to test whether the observed events are consistent with the signal-plus-background or the background-only hypotheses.

The uncertainty on the background shape is estimated from the uncertainties on the background fit parameters ( $a$, $b, c)$.

Given that the signal shape is based on simulation, we consider it to be affected by both experimental and theoretical uncertainties.

Experimentally, the signal may be affected by a number of sources. The integrated luminosity is known to a precision of $4.4 \%$ [9]. We generate the $M_{\mathrm{t}+\mathrm{g}}$ distribution for values of the jet energy scaled by \pm 1 standard deviation of the $\eta$ - and $p_{\mathrm{T}}$-dependent scale uncertainties from Ref. [10]. We account for the uncertainty related to energy resolution of jets by generating the $M_{\mathrm{t}+\mathrm{g}}$ distribution after adjusting the smearing by \pm 1 standard deviation (6-20\% depending on $\eta$ ). 
Further sources of experimental uncertainty include trigger efficiencies and lepton identification correction factors that are all obtained from data. The systematic uncertainty in the b-tagging efficiency is estimated by varying, one-by-one, the efficiency of tagging jets (from b- or cjets) and the mis-tag rate (from light-flavor jets) by \pm 1 standard deviation $[11,12]$. The systematic uncertainty due to the modeling of pileup events is checked by varying the average number of pile-up events by $\pm 4.4 \%$.

We estimate the effect of theoretical uncertainties from the PDFs by varying the CTEQ PDF parameters.

\section{Limit Calculation}

We examine the top plus jet mass spectrum for signs of a $t^{*}$ quark resonance and compute an upper bound on the $\mathrm{t}^{*} \mathrm{t}^{*}$ production cross section using Bayesian statistics [13]. The systematic uncertainties on the signal are modeled by nuisance parameters with log-normal priors. The background function plus signal template are used in a fit to the data using the negative log likelihood as a test statistic. The uncertainty on the background shape is incorporated by marginalizing the background-fit parameters using uniform priors. To combine the results from the $\mu+$ jets and e+jets channels, we multiply the likelihoods from the two channels together.

\section{Results}

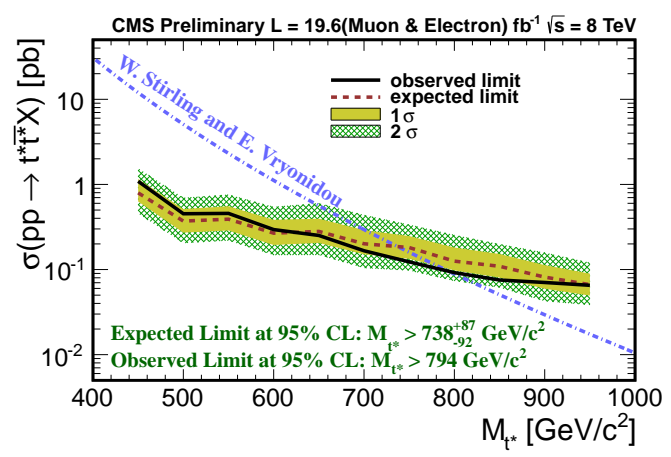

Figure 2. The observed (solid line) and expected (dashed line) 95\% CL upper limits for the $\mathrm{t}^{*} \mathrm{t}^{*}$ production cross section as a function of the $t^{*}$ mass for combined data. The \pm 1 and \pm 2 standard deviation ranges for the expected limits are shown by the bands. The theoretical cross section is shown by the dasheddotted line.

Figure 2 shows the observed and expected upper limits, at $95 \% \mathrm{CL}$, for the $\mathrm{t}^{*} \mathrm{t}^{*}$ production cross section as a function of the $t^{*}$ mass. The lower limit for the $t^{*}$ mass is given by the value at which the upper limit curve for the $\mathrm{t}^{*} \mathrm{t}^{*}$ production cross section intersects the spin3/2 RS theoretical curve from Ref. [3]. This gives the
95\% CL observed (expected) limit for the $\mathrm{t}^{*}$ mass of $790 \mathrm{GeV} / c^{2}\left(738_{-92}^{+87} \mathrm{GeV} / c^{2}\right)$.

\section{Summary}

We have conducted a search for excited top quarks that are pair produced in pp interactions and decay exclusively to a standard model top quark and a gluon. Events that have an electron or a muon and at least six jets, one of which is identified as a b-jet, are selected. A kinematic fit assuming $\mathrm{t}^{*} \mathrm{t}^{*}$ production is performed and a candidate $\mathrm{t}^{*}$ mass is reconstructed for every event. The reconstructed mass has been analyzed in the full $\sqrt{s}=8 \mathrm{TeV}$ proton-proton data sample, corresponding to an integrated luminosity of $19.6 \mathrm{fb}^{-1}$, and no significant deviations from the standard model predictions have been found. In the absence of signs of new physics, upper limits on the production of $\mathrm{t}^{*} \mathrm{t}^{*}$ are set as a function of the $t^{*}$ mass. By comparing the results to the predicted cross section for $\mathrm{t}^{*} \mathrm{t}^{*}$ production in the spin-3/2 RS model, we exclude masses below $790 \mathrm{GeV} / c^{2}$ at $95 \% \mathrm{CL}$.

\section{References}

[1] J. Beringer, J.F. Arguin, R.M. Barnett, K. Copic, O. Dahl, D.E. Groom, C.J. Lin, J. Lys, H. Murayama, C.G. Wohl et al. (Particle Data Group), Phys. Rev. D 86, 010001 (2012)

[2] B. Hassanain, J. March-Russell, J. Rosa, JHEP 0907, 077 (2009), 0904 . 4108

[3] W. Stirling, E. Vryonidou, JHEP 1201, 055 (2012), cross sections at $\sqrt{s}=8 \mathrm{TeV}$ obtained though private communication., 1110.1565

[4] W. Rarita, J. Schwinger, Phys.Rev. 60, 61 (1941)

[5] S. Chatrchyan et al. (CMS), JINST 03, S08004 (2008)

[6] J. Alwall, M. Herquet, F. Maltoni, O. Mattelaer, T. Stelzer, JHEP 1106, 128 (2011), 1106.0522

[7] J. Pumplin, D.R. Stump, J. Huston, H.L. Lai, P. Nadolsky, W.K. Tung, Journal of High Energy Physics 2002, 012 (2002)

[8] S. Agostinelli et al. (GEANT4), Nucl. Instrum. Meth. A506, 250 (2003)

[9] CMS Collaboration (CMS), CMS Physics Analysis Summary CMS-PAS-SMP-12-008 (2012), http: //cdsweb. cern. ch/record/1434360

[10] S. Chatrchyan et al. (CMS), J. Instrum. 6, P11002 (2011)

[11] CMS Collaboration (CMS), CMS Physics Analysis Summary CMS-PAS-BTV-11-004 (2011)

[12] CMS Collaboration (CMS), CMS Physics Analysis Summary CMS-PAS-BTV-11-003 (2011)

[13] G. Cowan, J. Phys. G37, 075021 (2010) 